\title{
La promoción de la investigación científica a través de la capacitación metodológica y técnica
}

Leticia Salomón*

Edwin Medina**

Jorge Amador**

\section{LAS EXIGENCIAS DE INVESTIGACIÓN CIENTÍFICA EN LAS INSTITUCIONES DE EDUCACIÓN SUPERIOR (IES)}

El desarrollo de la investigación científica en las IES está vinculada a los investigadores, los profesores y los estudiantes, lo que nos obliga a considerar su abordaje en dos instancias diferenciadas: por un lado, a nivel de los estudiantes de grado y posgrado, y, por otro, a nivel de los profesores/ investigadores que trabajan en los mismos niveles (grado y posgrado).

En la primera instancia, referida a los estudiantes, conviene precisar que los niveles de exigencia y calidad en su formación, son directamente proporcionales al grado académico en el que se insertan. Por ejemplo, a nivel de licenciatura el nivel de exigencia es mínimo, esperando que los estudiantes del nivel sean capaces de manejar principios metodológicos básicos y las técnicas mínimas que les permitan identificar un problema de investigación, diseñar una estrategia de abordaje y presentar los resultados con un mínimo de rigurosidad. Esto supone un esfuerzo doble que implica, por un lado, remediar la deficiencia y deformación investigativa con que ingresan los estudiantes a las IES (escasos conocimientos sobré qué hacer para recoger y sistematizar información primaria y secundaria; incorrecta utilización y citación de ideas y datos de otros autores; y muy poca capacidad para presentar resultados de forma sencilla y estructurada) y, por otro, proporcionar los conocimientos y generar las prácticas adecuadas para que aprenda a hacer investigación con un mínimo de exigencia de calidad a lo largo de su carrera en la universidad. A nivel de maestría, el nivel de exigencia de capacidad investigativa es más alto porque este nivel sigue siendo formativo aunque con un fuerte grado de investigación; los retos se presentan también a nivel remedial con los estudiantes que ingresan y, a nivel avanzado, con el manejo de métodos y técnicas básicas que

\footnotetext{
¿eticia Salomón, Dirección de Investigación Científica, investigacionunah.directora.Is@gmail.com

Edwin Medina, Dirección de Investigación Científica, Departamento de Proyectos de Investigación. investigacionunah.proyectos.em@gmail.com

Jorge Amador, Dirección de Investigación Científica, Departamento de Gestión y Cooperación, investigacionunah.gestion.ja@gmail.com
} 
les permitan realizar trabajos de calidad en las distintas asignaturas y en la elaboración y desarrollo de su trabajo de tesis. A nivel de doctorado y mucho más a nivel de postdoctorado, la situación se revierte: el componente esencial es investigación, con toda la rigurosidad teórica, metodológica y técnica, y el componente secundario es la formación. Los estudiantes de cada nivel deben ir fortaleciendo y demostrando de manera creciente sus capacidades en investigación, el rigor del método, la precisión de las técnicas y la presentación precisa de sus resultados. Y esto deben hacerlo con el sustento de un eje curricular en investigación en cuyos componentes (asignaturas, talleres o seminarios) vayan adquiriendo el conocimiento y las habilidades necesarias para hacer investigación de calidad en el área científica de su competencia. El acompañamiento del proceso por parte de profesores que sean investigadores es fundamental para que la investigación se traduzca en práctica constante y natural que haga de la misma un proceso estimulante, responsable y comprometido con el desarrollo de la ciencia y la satisfacción de las necesidades nacionales. Manejar el eje curricular de investigación como una reproducción de la docencia tradicional en la que el estudiante lee, memoriza y responde a preguntas de rigor sin el acompañamiento de una práctica dirigida que le haga "aprender a investigar investigando", es un error que produce un doble efecto negativo: reduce el impacto del eje curricular de investigación en su formación profesional y minimiza, si no es que elimina, el aporte de su carrera al desarrollo de las prioridades de la ciencia, la tecnología y la propia sociedad.

En la segunda instancia, la de los profesores/ investigadores que trabajan a nivel de grado y posgrado, la situación es un tanto diferente. Para empezar, es importante precisar que los que hacen investigación científica en una IES y los que responden por la cantidad y calidad de la misma, son los profesores de la universidad y no los estudiantes de la misma, porque estos son aprendices en tanto se encuentran en un proceso de formación profesional que supone la adquisición creciente del conocimiento y manejo teórico de su campo del conocimiento. Pueden y deben hacer investigación con el acompañamiento lasesoría de un investigador que puede actuar como profesor de una asignatura, como director de tesis o como mentor en un proceso formativo de investigadores. Los profesores que investigan pueden trabajar a nivel de grado o de posgrado, pero se convierten en el centro del quehacer investigativo cuando se interesan en la investigación científica y cuentan con las capacidades mínimas para hacerlo con rigurosidad científica, metodológica y técnica. De ellos y de sus habilidades depende su fortalecimiento como investigadores científicos, más allá del nivel académico en donde trabajen. De su visibilidad como investigadores en congresos, conferencias, talleres, simposios y foros, y de su perfil como autores de artículos científicos en revistas nacionales e 
internacionales, dependerá su reconocimiento personal e institucional como investigadores.

Estos profesores investigadores pueden ubicarse en cualquier escala académica (licenciatura, especialidad, maestría, doctorado o posdoctorado) o en cualquier categoría docente (desde la más baja a la más alta), trabajar a nivel individual 0 grupal, a nivel disciplinario, inter o multidisciplinario y publicar a nivel nacional 0 internacional, todos ellos son investigadores científicos si poseen la profundidad de conocimientos, la rigurosidad del método, la precisión de las técnicas, la novedad y pertinencia de sus temas de investigación y su profundo compromiso con la ciencia, la tecnología y la sociedad que demanda más y mejores conocimientos. No obstante, el desarrollo de las comunicaciones, la eliminación de fronteras del conocimiento, la apertura de las universidades al mundo y la universalidad de la ciencia, asociada a la importancia de la interdisciplinariedad, obligan a los investigadores a ampliar sus horizontes, superar la visión limitada de su escritorio 0 laboratorio y conocer lo que ocurre en otras partes del mundo en los temas que están investigando, en asociación con investigadores de otras disciplinas, universidades y países.

El desarrollo de la investigación científica requiere manejo teórico de una disciplina, lo cual se adquiere por la adquisición de grados académicos desde las licenciaturas hasta las especialidades, maestrías, doctorados y posdoctorados, o por una sabia combinación de alguno de ellos con la profundización personal del conocimiento en un tema específico. Pero también requiere manejo de los métodos y las técnicas cuantitativas y cualitativas que vuelvan rigurosos, convincentes, válidos y aceptables los resultados de un proceso de investigación; esto se adquiere parcialmente dentro del proceso formativo o complementariamente por una vía alternativa que permite la adquisición de conocimiento técnico específico, como es la capacitación en investigación e, inclusive, la capacitación en gestión de la investigación.

La capacitación se convierte en un instrumento indispensable para fortalecer las habilidades de los investigadores en campos que no conocieron en su proceso formativo o que olvidaron por falta de uso o por el tiempo transcurrido desde que finalizaron sus estudios, pero también se convierte en un instrumento central para fortalecer los conocimientos y las habilidades de los profesores que enseñan a investigar - a nivel de grado o posgrado- para que aumenten la calidad de la enseñanza de la investigación y puedan estimular, desarrollar y potenciar el interés y las habilidades estudiantiles en el ámbito de la investigación científica. 


\section{LACAPACITACIÓN Y LA CALIDAD DE LAINVESTIGACIÓN CIENTÍFICA}

En este apartado analizaremos porqué en el contexto de la educación superior se vuelve necesario implementar estrategias de capacitación que coadyuven el fortalecimiento de uno de los tres ejes fundamentales de la vida universitaria actual: la investigación científica.

Al hablar de capacitación en investigación se hace referencia, por lo general, a cursos, talleres o diplomados sobre metodología de investigación, que comprenden aspectos estructurales del proceso y que pueden incluir desde temas epistemológicos hasta asuntos operativos de la investigación, como calcular una muestra, elaborar instrumentos de recolección de datos o redactar informes de investigación. Se incluye, además, la formación recibida en programas de estudio de nivel superior a través de especialidades, maestrías y doctorados.

\section{¿Por qué Capacitar en Investigación?}

En América Latina, el apoyo brindado a la educación en general y a la educación superior en particular, durante la década de los años ochenta, fue relativamente bajo y se caracterizó por una reducción de los recursos económicos de apoyo a la Instituciones de Educación Superior (IES) justificada en el incremento del gasto en defensa y seguridad o el fortalecimiento de los ejércitos nacionales (Gazzola, A. y Didriksson, $A, 2008)$. Esto redundó en una educación superior que enfatizaba la docencia sobre la investigación y la extensión, generando nuevos profesionales para el mercado laboral pero generando muy poco conocimiento científico 0 brindando un bajo aporte a la solución de los problemas nacionales.

Al reducirse el conflicto bélico derivado de la guerra fría, a partir de los años noventa, con el advenimiento del cese de la guerra fría, la región latinoamericana se vio en la necesidad de ajustar sus políticas para formar parte y manejar las relaciones en el nuevo mundo globalizado. Estos ajustes tienen que ver también con la manera de mejorar su economía y desarrollo humano, lo que hace que se replantee el rol de las universidades como actores clave en la generación y transmisión de dicho conocimiento.

Algunos analistas comparan este volver a la universidad, con el cambio que se dio de la universidad napoleónica a la universidad humboltiana que incorporó la ciencia y la investigación como parte fundamental de su quehacer. A partir de entonces las universidades han estado llevando a cabo acciones de modificación de sus procesos formativos y de los contenidos de sus mallas curriculares y han avanzado 
en la definición del rol que las instituciones universitarias deben cumplir en el desarrollo de los países. (Santelis, 2010)

En el marco de la globalización, la era de la información y la inminente internacionalización de la educación superior, las universidades se volvieron gestoras o depositarias de mayores recursos para realizar investigación en base a estándares internacionales y nacionales cada vez más exigentes, que demandan de ellas calidad, transparencia en el financiamiento, rendición de cuentas, flexibilidad curricular, equidad y pertinencia, uso y manejo de tecnologías de información y comunicación y transferencia de nuevos conocimientos enmarcados dentro de nuevos ordenamientos jurídicos, legislativos, políticos y organizacionales, aunados a procesos de investigación que deben contar con un fuerte respaldo científico y metodológico, con el que no se cuenta en la mayoría de las universidades de América Latina, por lo que vuelve necesaria la capacitación permanente para lograr fortalecer la investigación (Gazzola, A. y Didriksson, A, 2008), entendiéndose ésta, la capacitación, como una de las muchas estrategias que se pueden implementar para desarrollar la investigación universitaria pues se sabe que la investigación científica es una actividad que se aprende haciendo. Es importante destacar, en este sentido, que los responsables de la investigación en América Latina han sido, y siguen siendo, las universidades públicas, mismas que están trabajando en de distintos procesos de reforma, por mantenerse al día con las demandas de la globalización y la sociedad del conocimiento.

\section{¿En qué y cómo capacitar?}

La realización de investigación con la rigurosidad científica necesaria sigue siendo un reto para las instituciones de educación superior y en particular para los docentes que desean iniciarse en esta actividad pues el proceso de investigación le parece muy complejo y riguroso al investigador novato, quien en muchas ocasiones se desanima ante las exigencias metodológicas, técnicas y el cumplimiento de cronogramas de trabajo y entrega de informes. Es por esta razón que se vuelve necesario capacitar en estos temas por medio de estrategias que involucren tanto los aspectos teóricos como operativos que permitan al investigador novato tener una experiencia hands on guiada por especialistas en cada rama y que al mismo tiempo le brinden el apoyo técnico y metodológico en las diferentes fases del proceso de investigación. En este sentido toma preponderancia capacitar a los docentes investigadores en la elaboración de un diseño o protocolo de investigación que oriente todo el proceso y que establezca con claridad los objetivos y el alcance de la investigación en relación con los fondos y resultados esperados. La elaboración de un protocolo o diseño es una actividad fundamental en el proceso de 
investigación y poderlo elaborar es una competencia clave que deben poseer los profesores pues tanto a nivel de las universidades nacionales e internacionales como de organismos cooperantes y fuentes de financiamiento, es un requisito fundamental para determinar la asignación de recursos a un proyecto de investigación, brinda una visión amplia de todas las fases del proyecto y permite saber con antelación de qué recursos (humanos, financieros, materiales, etc.) tendrá necesidad para poder culminar exitosamente la investigación.

La calidad de los instrumentos y técnicas de recolección está directamente relacionada con la calidad de los datos que se puedan obtener en el estudio y consecuentemente con los resultados que se presentan. La posible generalización o transferibilidad de estos resultados está también relacionada con el adecuado cálculo muestral por lo que se considera fundamental enfatizar en estos componentes del diseño de investigación dentro de un programa de capacitación en metodología de la investigación.

Otro aspecto que es fundamental fortalecer es el análisis estadístico de los datos pues la mayoría de investigaciones que se realizan en la universidad, principalmente desde las ciencias sociales, se quedan a nivel descriptivo sin aprovechar las potencialidades que brinda la estadística inferencial para el cálculo de probabilidades, de modo que se puedan hacer inferencias de manera responsable para facilitar la toma de decisiones, con riesgo mínimo de error, sobre los distintos problemas que se investigan. (Amón, 1980)

En muchos casos, los resultados de las investigaciones, en las que se ha invertido tiempo y recursos financieros y humanos de la universidad, son desestimados precisamente por errores técnicos que se pueden evitar o subsanar por medio de procesos de capacitación-acción, es decir procesos que involucren un conocimiento teórico pero con un fuerte componente práctico que permita a los investigadores apropiarse de la metodología y utilizarla adecuadamente.

Si bien es cierto la metodología de investigación es un aspecto fundamental para el desarrollo de proyectos y estudios en la universidad, no se puede dejar de lado el aspecto de la gestión y administración de recursos para investigación. En muchos casos los investigadores han logrado un manejo eficiente de los aspectos metodológicos de la investigación pero no así de los aspectos administrativos y de gestión. Esto les impide o limita su participación en proyectos de investigación a nivel regional o internacional debido a lo engorroso que pueden ser los formularios, requisitos y demás que se deben cumplir para obtener fondos de proyectos internacionales, de la Unión Europea o de universidades o instituciones que 
promueven la investigación en las universidades de América Latina. Esto ha obligado, en muchos casos, a que algunas universidades participen únicamente como "socios" de redes internacionales de investigación 0 en proyectos coordinados por otras universidades que cuentan con el personal humano capacitado en la gestión de fondos para investigación. En este sentido, además de contar con una capacitación sobre los aspectos administrativos generales de un proyecto de investigación, se debería contar en cada universidad con un grupo especialmente capacitado en la gestión de recursos y elaboración y presentación de propuestas y proyectos para concursar por fondos internacionales y que sean también el pilar de apoyo para los investigadores de la universidad en los aspectos meramente administrativos, de manera que los investigadores se puedan dedicar a realizar la investigación propiamente dicha. En tanto no se logre tener este grupo especializado de gestores, se deberá continuar capacitando a los docentes también en cómo llevar a cabo la administración y seguimiento efectivos de los proyectos de investigación.

Un aspecto que resulta fundamental para que los procesos de capacitación tengan un impacto duradero en las universidades tiene que ver con el hecho de que estas capacitaciones y todos los aspectos que en ellas se trabajen, estén relacionados directamente con las prioridades de investigación de las universidades, de manera que los esfuerzos se coordinen desde todas las áreas, consecuentes con los intereses y prioridades de las universidades y que los profesores que se están capacitando vayan adquiriendo una visión más amplia de hacia dónde se deben orientar las futuras investigaciones.

Por último y dada la importancia que tiene para las universidades la publicación de los resultados de las investigaciones, pues es uno de los indicadores clave para la clasificación de las universidades en los ranking internacionales, se debería promover la capacitación tendente a la escritura de artículos científicos considerando las exigencias relacionadas con el tipo de revista en que será publicado (primer cuartil, segundo cuartil, etc.), considerando las exigencias de cada una de las mismas y explicando el proceso que conlleva la publicación de un artículo científico en una revista indexada y que pasa por una revisión rigurosa hasta llegar a la eventual publicación en caso de que el artículo sea aceptado.

A mediano y largo plazo se percibe a la capacitación como un elemento fundamental en la promoción de la investigación universitaria que conlleve a la generación de una masa crítica de investigadores que puedan dar respuesta a los problemas sociales que encaran los diferentes países y las mismas universidades e insertarse adecuadamente en un mundo globalizado. La investigación científica, además, 
debe aportar resultados que se puedan integrar con la docencia y la vinculación, de manera que exista una complementariedad entre las tres funciones de la universidad y en la que cada una apoye y retroalimente a las otras, de tal manera que, al promover la capacitación en investigación científica, se promuevan reformas en el modo pensar y no se queden a nivel de ajustes orientados a responder operativamente a demandas puntuales sino que conlleven la integración de la docencia, la investigación y la vinculación en el seno de la universidad. Esta es la realidad que las universidades latinoamericanas deben considerar y enfrentar.

\section{LA CAPACITACIÓN Y LA CALIDAD DE LA ENSEÑANZA DE LA INVESTIGACIÓN CIENTIIFICA}

Las Universidades se han globalizado, el conocimiento está latente, las necesidades sociales miran hacia éstas para buscar respuestas dentro de la academia y superar ese rol tradicional de formadoras de profesionales sin un papel claro en la producción y transferencia del conocimiento.

Las Normas Académicas del Nivel Superior definen a la Universidad como responsable de una pluralidad de áreas, campos y programas académicos, siendo sus objetivos; a) impartir enseñanza, en todas las ramas de las ciencias; b) contribuir al progreso de la ciencia, a la formación de investigadores y al desarrollo de la investigación científica, literaria y técnica, c) preparar a los futuros profesionales, exigiéndoles a su vez un amplio y cualitativo acervo de conocimientos específicos, acordes con el rol que van a desempeñar en la sociedad, y ch) proporcionar una cultura superior y un perfeccionamiento personal y profesional necesarios para asimilar los avances científicos y responder cualitativamente a la demanda social.

En el contexto anterior debemos preguntarnos si en nuestras universidades tenemos profesores capacitados para enseñar a investigar y si tenemos profesores investigadores que podrían formar a los futuros investigadores en las diversas áreas del conocimiento y así procurar ser responsables de la tarea que el Estado y la misma sociedad requieren.

La concepción de la enseñanza de la investigación en grados y postgrados y el rol del profesor

De forma tradicional se ha enseñado la investigación como una asignatura sin relevancia; la mayoría de los estudiantes la cursan y no existen aprendizajes significativos; se considera la investigación como algo ajeno y, peor aún, de forma 
aislada de la formación de profesionales.

En las carreras de grado, la investigación es una asignatura en la etapa terminal de la formación, en la que el profesor no puede hacer una conexión entre la metodología de la investigación y los problemas que se pueden suscitar en su campo de especialización, programando como parte práctica de la misma, "vamos a investigar" sin orientar apropiadamente acerca de qué investigar, cómo hacerlo y cómo presentarlo, perdiendo la oportunidad de impulsar y darle sentido a esa metodología, que en la mayoría de los casos es asumida como algo complejo y de difícil aplicación.

El caso de los programas de postgrados no es diferente, se incluye algunas veces y de forma forzada un módulo de "metodología de la investigación" o un "taller de tesis", en los que, por lo general, no se desarrollan más que los objetivos y el contenido, evidenciando el aprendizaje a través de formas conductistas (exámenes, test y guías de estudio, etc.) siendo teóricas, memorísticas y repetitivas, y sin servir de aplicación para que estos estudiantes realmente investiguen y lo hagan con calidad.

Nuestras universidades tradicionalmente se han dedicado al desarrollo de los "contenidos", centradas casi exclusivamente en la docencia; es por ello que los profesores sólo se han encargado de ello, de ser profesores dedicados exclusivamente a la docencia con poca o ninguna vinculación con las otras funciones de la universidad. Las universidades tienen como función básica el desarrollo de la docencia, investigación y vinculación, lo que obliga a los docentes a capacitarse para ejercer en esas tres funciones.

Debemos preguntarnos si nuestras universidades necesitan de profesores investigadores para que enseñen a investigar o sólo es necesario un profesor que desarrolle contenidos de metodología de la investigación.

Es indudable que un profesor con experiencia en investigación cuenta, además del conocimiento sobre la metodología, con el valor agregado de la experiencia, lo que significa que sus estudiantes tendrán una visión amplia del proceso de investigación y no sólo la versión de que repite lo que está contenido en el libro. Lo ideal sería que esos profesores, que con entusiasmo imparten las asignaturas de investigación en las carreras de grado y módulos en los postgrados, contaran con espacios y oportunidades para desarrollar investigaciones que vuelvan práctico ese conocimiento ganado en los procesos de capacitación. 
La promoción de la capacitación en investigación como factor de mejora de la calidad de la enseñanza y los procesos de formación permanente.

Un ideal en las universidades es la mejora de la calidad de la enseñanza, teniendo como tendencia una mejora en el aprendizaje que se exprese en una mejor formación profesional del estudiantado; la pregunta que se hace al respecto es ¿qué factores o estrategias se pueden establecer para lograr esa mejora? Una de esas respuestas es procurar que los profesores constantemente se capaciten en procesos que tengan dos grandes objetivos: el primero, lograr la actualización sobre los aprendizajes de investigación logrados en una etapa de su formación profesional y, la segunda, la formación y consolidación del dominio de la metodología de la investigación de la cual carecen estos profesores; por lo tanto, la capacitación en investigación cumple una doble función referida a la formación inicial y a la formación permanente de investigadores y profesores que enseñan a investigar.

El resultado final de los procesos de capacitación, en especial en investigación, es volver competente al profesor universitario para que enseñe a investigar, y no sólo sea un desarrollador de contenidos de la metodología de la investigación; que éste haga del poco espacio que se destina en nuestras universidades a la investigación, un ámbito especializado de formación de competencias investigativas.

Tunnermann (2003:71), en "La Universidad ante los retos del Siglo XXl", plantea que la respuesta de las universidades ante los desafíos contemporáneos, es su compromiso con la educación permanente, y, además, arguye que "es urgente estructurar las respuestas de las universidades, mediante una serie de tareas, que en apretada síntesis conducirian a fortalecer sus capacidades de docencia, investigación y extensión interdisciplinarias; flexibilizar sus estructuras académicas e introducir en su quehacer el paradigma del aprendizaje permanente; auspiciar sólidos y amplios programas de actualización y superación académica de su personal docente, acompañados de los estímulos laborales apropiados".

El riesgo que los profesores universitarios tienen con respecto a la formación inicial que tuvieron en investigación (la adquirida cuando obtuvieron el grado académico) es que muchos pretenden enseñar cómo se les enseñó, creyendo que el conocimiento se congeló en el tiempo y, aun con mayor riesgo, el pensar que todo sobre investigación ya fue aprendido por él y no hay nada más sobre lo que no tenga dominio.

Ante esto Phillip Coombs (1991:54) sostiene que en el futuro los títulos 
profesionales no serán más que un certificado de que el graduado aprendió a aprender. Y otro analista va más allá cuando sostiene que hacia el año 2030, los títulos profesionales llevarán un sello que dirá expira en diez años, como las medicinas de las farmacias.

Así también las Normas Académicas del Nivel Superior consideran como un deber y a la vez un derecho del profesor universitario "participar en los programas de capacitación que promueva cada centro y el nivel, para renovar constantemente su formación profesional y pedagógica" y "ser candidato a programas de formación y capacitación, dentro o fuera del país, con el patrocinio de cada centro educativo o el nivel".

La mejora de la calidad de la enseñanza en la investigación a través de la capacitación. Balance de los resultados.

Desde una relación de costo - beneficio sobre los esfuerzos de nuestras universidades por promover procesos de capacitación en investigación, tendremos que alcanzar estos resultados:

- Profesionales con competencias investigativas para resolver problemas de la sociedad: al lograr aprendizajes significativos en los estudiantes sobre investigación tendremos profesionales formados con iniciativas para que investiguen problemas sociales proponiendo soluciones científicas.

- Profesionales que desarrollen ciencia desde sus campos del conocimiento: tendremos profesionales formados con competencias investigativas básicas y específicas para hacer "ciencia", entendida que todas las áreas del conocimiento la hacen aportando estos resultados al mundo que día con día requiere más de ella, desarrollando proyectos de investigación.

- Profesores investigadores universitarios formando profesionales de alta calidad: si nuestras universidades propiciaran a nuestros profesores que enseñan a investigar espacios y oportunidades para que también investiguen, no quedaría en el desarrollo de un simple contenido, sino que los guiarían a través de experiencias de aprendizaje para que al insertarse en el campo laboral estos se sientan habituados a resolver problemas sociales cotidianos.

- Universidades generadoras de conocimiento: al hacer de la enseñanza de la investigación algo práctico, cotidiano y necesario tendremos la generación de nuevo conocimiento, y será tan necesario como el hecho de respirar, dejar a un lado que investigar es algo difícil y que sólo lo hacen los científicos, para convertirlo en una práctica general y consustancial al ejercicio de la docencia.

- Programas de capacitación flexibles, cambiantes y diversos: como proceso 
innovador, es difícil entrar, pero a medida que los profesores internalicen que es necesario seguir formándose permanentemente (capacitación continua), la demanda de la misma irá aumentando a la vez que obligará a diversificar la oferta en su forma de entrega (semipresencial, virtual, etc.), y a ser más específica, de acuerdo a las necesidades derivadas del desarrollo tecnológico y de las exigencias metodológicas de la sociedad del conocimiento.

\section{REFERENCIAS}

Amon, J. (1980) Estadística para Psicólogos, Editorial Pirámide, España Coombs, Philip (1991), "Universidad e investigación", en Asterisco Cultural, Madrid, N.4.

Gazzola, A, y Didriksson, A. (2008) Tendencias de la Educación Superior en América Latina y el Caribe. IESALC-UNESCO, Caracas, Venezuela.

Santelis, B. (2010) Informe sobre Educación Superior Iberoamericana, Centro Interuniversitario de Desarrollo (CINDA), Santiago, Chile.

Tunnermann, B.C. (2003). La Universidad ante los retos del Siglo XXI, México: Universidad Autónoma de Yucatán. 